\title{
Le pèlerinage contemporain en sciences sociales : moments, bifurcations, nouveaux horizons
}

\section{Rémy Délage}

\section{(2) OpenEdition \\ 1 Journals}

Édition électronique

URL : https://journals.openedition.org/assr/29730

DOI : $10.4000 /$ assr.29730

ISSN : $1777-5825$

Éditeur

Éditions de l'EHESS

Édition imprimée

Date de publication : 1 décembre 2017

Pagination : 155-167

ISSN : 0335-5985

\section{Référence électronique}

Rémy Délage, «Le pèlerinage contemporain en sciences sociales : moments, bifurcations, nouveaux horizons ", Archives de sciences sociales des religions [En ligne], 180 | octobre-décembre 2017, mis en ligne le 01 décembre 2019, consulté le 22 septembre 2021. URL : http://journals.openedition.org/assr/ 29730 ; DOI : https://doi.org/10.4000/assr.29730 


\title{
Rémy Delage
}

\section{Le pèlerinage contemporain en sciences sociales Moments, bifurcations, nouveaux horizons}

\author{
À propos de: \\ Albera Dionigi, EAde John (eds.), International Perspectives on Pilgri- \\ mage Studies. Itineraries, Gaps and Obstacles, Londres, Routledge, \\ 2015, 213 p. \\ Albera Dionigi, Eade John (eds.), New Pathways in Pilgrimage Studies. \\ Global Perspectives, Londres, Routledge, 2017, 216 p.
}

Deux éminents spécialistes de l'étude du pèlerinage, le Britannique John Eade et l'Italien Dionigi Albera, basés respectivement en Angleterre et en France, s'allient dans ce projet de publication à deux volets complémentaires, dont l'objectif principal est de libérer les pilgrimage studies de l'hégémonie anglo-saxonne ${ }^{1}$. En effet, à l'évocation du phénomène pérégrin comme objet d'étude, nous pensons inévitablement à l'influence majeure jouée par Edith et Victor Turner qui avaient formulé de nouveaux paradigmes. Un champ de recherche relativement autonome sur les pèlerinages s'est ainsi constitué à partir des années 1970-1980, en Amérique du Nord et en Europe, au sein des sciences sociales des religions anglophones. Cependant, plusieurs auteurs majeurs comme Michael Sallnow, John Eade ou Simon Coleman critiquent la théorie turnerienne de la communitas et en dénoncent les principales apories. Plus ou moins en phase avec les grands tournants ou virages épistémologiques (notamment culturel, spatial, mobilitaire) chers aux sciences sociales anglo-saxonnes, ces auteurs publient deux ouvrages qui feront date, l'un centré sur l'idée de "contestation ", voyant le pèlerinage comme une arène à l'intérieur de laquelle s'affrontent des discours contradictoires, révélatrice de conflits de représentation du religieux ${ }^{2}$, et l'autre posant le «mouvement» comme force structurant les multiples dimensions du pèlerinage ${ }^{3}$.

1. I. Reader, «Breaking Barriers, Eroding Hegemony: Reflections on the Importance of Multilingual Studies of Pilgrimage beyond the Anglophone World ", in Albera D., Eade J. (eds.), New Pathways in Pilgrimage Studies. Global Perspectives, Londres, Routledge, 2017, p. 181-198.

2. J. Eade, M. Sallnow (eds.), Contesting the Sacred: The Anthropology of Christian Pilgrimage, Londres/New York, Routledge, 1991.

3. S. Coleman, J. Eade (eds.), Reframing Pilgrimage: Cultures in Motion, Londres/New York, Routledge, 2004. 
Ces changements de paradigme constituent autant de moments de bifurcation importants dans la carrière de l'objet «pèlerinage» en sciences sociales du religieux. Aussi Simon Coleman invitait-il les pèlerinologues, dès le début des années 2000, à se décentrer de leur objet essentiellement appréhendé dans sa dimension religieuse et à partir de postures épistémologiques émanant quasi-exclusivement de leur propre champ de savoir. Pour l'auteur, il convenait alors d'élargir les horizons ethnographiques, en convoquant d'autres échelles de comparaison en dehors de la chrétienté occidentale ${ }^{4}$ et du champ autonome des études du pèlerinage.

Si ce renouvellement des approches a bien été intégré au-delà de la sphère académique anglo-saxonne, qu'en est-il de l'apport d'autres écoles de pensée non-anglophones mais aussi non-occidentales à l'analyse du pèlerinage en sciences sociales? Ces travaux sont longtemps restés, et le sont toujours pour certains d'entre eux, inaccessibles aux anglo-saxons à cause de la barrière linguistique. L'objectif de Dionigi Albera et John Eade est donc de combler partiellement cette lacune en offrant à un lectorat anglophone l'opportunité de découvrir d'autres approches et recherches conduites à travers le monde. Inversement, la lecture cumulée de ces ouvrages est tout aussi stimulante pour les autres chercheurs, non-anglophones, trop souvent enfermés dans leurs zones de confort, disciplinaires ou en études aréales. N'est-il pas ironique de constater que les contributions réunies dans le volume International Perspectives ne concernent que l'Eurasie (de la France au Japon et à la Russie) et le christianisme (surtout le catholicisme) ? Ce serait ne pas reconnaître en effet que les deux ouvrages se font écho et doivent s'appréhender comme un seul livre, le second volet couvrant justement les autres aires culturelles (de l'Afrique du Nord à l'Asie du Sud, du Mexique et du Brésil jusqu'à l'Australie et l'Afrique du Sud) et les religions non-chrétiennes (islam, bouddhisme, hindouisme, taoïsme).

En l'absence de découpage thématique, chronologique ou disciplinaire dans chacun des deux volumes, chaque contributeur se focalise sur une ou plusieurs traditions d'analyse en mettant en avant les grandes figures passées et présentes qui ont marqué les sciences sociales des religions et du pèlerinage, et ce dans les limites de leur contexte académique et géographique. Ils en relèvent également les lacunes et les contradictions, tout en suggérant de nouvelles pistes de recherche à explorer, de nouveaux terrains à défricher. Au final, ne s'agissant pas d'études thématiques individuelles, chacun des articles faisant la recension systématique de travaux anciens et d'approches plus ou moins novatrices, la lecture intégrale des deux ouvrages en devient parfois laborieuse et l'analyse comparative d'autant plus difficile que le lecteur ne peut être familier avec l'intégralité des cas présentés. Mais ce serait, encore une fois, ignorer un autre objectif de ce projet de publication, celui de proposer à un public étudiant un panorama global des approches du pèlerinage en sciences sociales.

4. Certains auteurs travaillant dans différentes régions du monde préfèrent utiliser la notion moins polémique de "voyage rituel », plutôt que celle de "pèlerinage ", dont l'origine chrétienne renvoie à une représentation dichotomique de l'espace entre le sacré et le profane, peu opérationnelle dans d'autres contextes. 


\section{Cartographie d'un champ de recherche}

Comme souligné dès l'introduction du premier volume ${ }^{5}$, les études de pèlerinage ne commencent pas avec les Turner et la communitas, une notion d'ailleurs contenue en germes dans nombre de travaux datés de la première moitié du $\mathrm{xx}^{\mathrm{e}}$ siècle (notamment Arnold Van Gennep en France), et ce même si le pèlerinage ne devient un objet de recherche à part entière au sein d'un champ des sciences sociales occidentales qu'à partir des années 1970-1980. La lecture comparée de l'ensemble des contributions permet d'une part, d'identifier les lieux de production des connaissances sur le pèlerinage à l'échelle mondiale et, d'autre part, de reconstruire à grands traits leur généalogie, ces savoirs étant historiquement situés.

C'est à la fin du $\mathrm{XIX}^{\mathrm{e}}$ et au début du $\mathrm{XX}^{\mathrm{e}}$ siècle que l'histoire commence véritablement avec l'émergence dans de nombreux pays du folklore comme champ académique pluridisciplinaire, plus ou moins autonome par rapport aux universités et aux institutions de recherche établies. Centrées sur l'étude de la société paysanne, des coutumes et de la religion populaire, en somme sur la religiosité paysanne par opposition aux formes institutionnalisées de la dévotion, comme ce fut le cas dans plusieurs pays européens, le pèlerinage n'était pas vraiment au centre des préoccupations, tout du moins dans sa double dimension rituelle et circulatoire. En effet, si dans le sillage de Paul Sébillot et d'Arnold Van Gennep (rites de passage), de nombreux chercheurs francophones se sont évertués à rechercher les survivances du paganisme dans les rituels populaires, Anna Fedele et Cyril Isnart ${ }^{6}$ nous rappellent qu'il a fallu attendre Robert Hertz (début $\mathrm{Xx}^{\mathrm{e}}$ siècle) pour disposer d'une première monographie, passée sous silence en dépit de son approche très innovante pour l'époque, consacrée à un site alpin de pèlerinage catholique.

On retrouve les mêmes approches dans plusieurs pays européens comme en Italie $^{7}$ où Giuseppe Pitrè a marqué de son sceau les études folkloriques centrées sur la religion populaire et les processions locales (plutôt que sur le pèlerinage), en Hongrie et en Pologne ${ }^{8}$, mais aussi en Allemagne et dans les territoires germanophones où l'accent était mis pendant très longtemps sur la culture matérielle du pèlerinage, que plusieurs musées ethnographiques permirent de valoriser auprès du grand public ${ }^{9}$. Au Japon, le folklore s'est lui constitué comme discipline à part

5. D. Albera, J. Eade, «International Perspectives on Pilgrimage Studies: Putting the Anglophone Contribution in Its Place", in Albera D., Eade J. (eds.), International Perspectives on Pilgrimage Studies. Itineraries, Gaps and Obstacles, Londres, Routledge, 2015, p. 14.

6. A. Fedele, C. Isnart, «From Cryptic to Critique: Early and Contemporary French Contributions to the Study of Pilgrimage ", in Albera D., Eade J. (eds.), International Perspectives on Pilgrimage Studies..., op. cit., p. 171-192.

7. E. Zapponi, «Italian Studies on Pilgrimage. Beyond Folklore, Towards a National Anthropological Tradition and the International Circulation of Ideas ", in Albera D., Eade J. (eds.), International Perspectives on Pilgrimage Studies..., op. cit., p. 152-170.

8. A. Niedźwiedź, "Old and New Paths of Polish Pilgrimages », in Albera D., Eade J. (eds.), International Perspectives on Pilgrimage Studies..., op. cit., p. 69-94 et G. Barna, "Pilgrimages in Hungary: Ethnological and Anthropological Approaches ", in Albera D., Eade J. (eds.), International Perspectives on Pilgrimage Studies..., op. cit., p. 95-113.

9. H. Eberhart, «From Religious Folklore Studies to Research of Popular Religiosity: Pilgrimage Studies in German-Speaking Europe », in Albera D., Eade J. (eds.), International Perspectives on Pilgrimage Studies..., op. cit., p. 114-133. 
entière dès le début du $\mathrm{xx}^{\mathrm{e}}$ siècle, distincte des études religieuses ou de l'anthropologie, celle-ci ne s'appliquant qu'à des terrains extérieurs à la société nippone ${ }^{10}$. L'un des dénominateurs communs à ces courants d'étude fut incontestablement, comme le relèvent justement Dionigi Albera et John Eade, de relier le pèlerinage au discours et à l'idéologie nationaliste. Parallèlement, en contexte colonial, que ce soit au Maghreb et au Moyen-Orient comme en Asie du Sud, ce sont les premiers orientalistes et ethnographes européens (voyageurs, officiers, espions-géographes) qui ont décrit les coutumes locales et les formes populaires de religiosité islamique. Il est intéressant de noter par exemple, avec Katia Boissevain, que les premières ethnographies datées du début du $\mathrm{Xx}^{\mathrm{e}}$ siècle voyaient dans les confréries soufies non pas seulement le témoignage d'un monde préislamique (recherche de survivances) mais aussi une forme de mobilisation et d'opposition à l'empire ottoman puis à l'occupation française ${ }^{11}$, près d'un siècle avant que le paradigme de la contestation ne soit formalisé et appliqué par les anthropologues à de nombreux cas d'études de pèlerinage dans le monde.

Il convient d'ajouter que le développement des champs de savoir est bien souvent tributaire des changements de régime politique et de l'orientation des politiques publiques en matière d'enseignement supérieur et de recherche. Alors que les études de pèlerinage se sont continuellement développées en Amérique du Nord puis en Europe occidentale durant la deuxième moitié du $\mathrm{xx}^{\mathrm{e}}$ siècle, il fallut attendre en revanche la chute du mur de Berlin et du communisme pour voir s'ouvrir de nouveaux espaces académiques et institutionnels. En effet, les sciences sociales du religieux furent quasi-inexistantes en Chine et dans l'Union soviétique ${ }^{12}$ pendant près d'un demi-siècle, les priorités de recherche étant essentiellement fixées par l'État et les élites dans des contextes où l'idéologie communiste prônait l'effacement des cultes populaires et dévotionnels au seul profit des cultes officiels, fortement régulés par le pouvoir central. Pareillement, dans un pays laïc comme le Mexique, les relations complexes entre l'État et l'institution catholique n'ont pas facilité le développement des recherches sur la « religion populaire » que seule l'Église encourageait; celles-ci traitaient plutôt la question de la sécularisation selon une perspective marxiste ${ }^{13}$. L'objet pèlerinage n'y est apparu qu'à partir des années 1980, dans le sillage des travaux fondateurs de Gimenez sur la religion populaire, l'identité et le territoire. Dans un tout autre contexte, comme au Japon, Ian Reader nous signale que les chercheurs japonais n'ont pris connaissance que très tardivement du bagage théorique de Victor et Edith Turner, soit seulement au début des années 2000, une situation liée à l'insularité académique du Japon, qui

10. I. Reader, "Japanese Studies of Pilgrimage", in Albera D., Eade J. (eds.), International Perspectives on Pilgrimage Studies..., op. cit., p. 23-46.

11. K. Boissevain, "Studying Religious Mobility: Pilgrimage, Shrine Visits and Religious Tourism from the Maghreb to the Middle East ", in Albera D., Eade J. (eds.), New Pathways in Pilgrimage Studies..., op. cit., p. 95.

12. M. Bingenheimer, "Pilgrimage in China ", in Albera D., Eade J. (eds.), New Pathways in Pilgrimage Studies..., op. cit., p. 18-35 et Stella Rock, "Touching the Holy: Orthodox Christian Pilgrimage within Russia », in Albera D., Eade J. (eds.), International Perspectives on Pilgrimage Studies..., op. cit., p. 47-68.

13. A. A. Ros, "Transcending Symbols: The Religious Landscape of Pilgrimage Studies in Mexico ", in Albera D., Eade J. (eds.), New Pathways in Pilgrimage Studies..., op. cit., p. 142-161. 
s'est en partie construite par opposition à l'européocentrisme des sciences sociales du pèlerinage ${ }^{14}$ mais qui s'est traduite par un nationalisme méthodologique très puissant jusqu'à aujourd'hui. Cette absence de dialogue avec les sciences sociales occidentales a néanmoins eu pour effet positif de voir se développer des approches innovantes sur l'agencement des lieux saints et des pèlerinages dans leurs rapports à l'environnement et à la société locale.

Dernier point, les changements de géopolitique régionale liés soit à la décolonisation, soit à l'effondrement de l'ex-URSS n'ont pas seulement permis aux travaux occidentaux (surtout les sciences sociales anglo-saxonnes, françaises et allemandes) de pénétrer ces nouveaux territoires eurasiatiques; ils ont également entraîné la réorganisation, à différentes échelles, des géographies religieuses ainsi que la revitalisation de la pratique pèlerine et la réactualisation d'anciennes routes, voire la création de nouveaux centres de pèlerinage. Longtemps orientées par le sentiment nationaliste, comme en Pologne où le tracé des frontières internationales a fortement évolué au cours des deux derniers siècles, période durant laquelle les idées de "pèlerinage national " puis de "pèlerinage patriotique " émergent au sein de la société paysanne ${ }^{15}$, les recherches sur la religiosité catholique à partir des années 1990 prennent pour échelle d'analyse le transnational et s'intéressent désormais aux non-catholiques et aux minorités religieuses. Toujours en Europe de l'Est, les réseaux de pèlerinage se sont réorganisés entre 1950 et 1989; d'anciennes routes de pèlerinage ont été coupées, traversant désormais les frontières internationales entre la Hongrie et ses voisins, donnant naissance à de nouveaux lieux de culte transnationaux ${ }^{16}$.

Ces quelques idées et exemples, que nous prenons ici pour représentatifs des principaux moments dans la structuration des savoirs académiques sur le pèlerinage, servent de jalons à une géographie des territoires intellectuels, déséquilibrée car fortement dominée par les théories anglo-saxonnes sur le pèlerinage. Or, ce que révèlent ces deux ouvrages, et c'est là leur principal enjeu, est une géographie multipolaire partagée aujourd'hui entre le monde anglo-saxon (de l'Amérique du Nord à l'Australie), l'Europe occidentale non anglophone dont surtout la France, l'Italie et l'Allemagne, des territoires académiques longtemps fermés pour des raisons linguistiques ou géopolitiques (Russie, Chine, ex-républiques soviétiques) mais aussi d'anciennes colonies au Maghreb et au Proche-Orient, en Afrique du Sud. Cet essai de cartographie dynamique des savoirs, même si le projet général de ces deux publications n'est pas présenté comme tel, montre une véritable diversité des lieux et des contextes de production de la connaissance; l'ouverture, temporaire ou durable, de frontières internationales associée à de nouveaux régimes de mobilité (cas des circulations migratoires à l'échelle transnationale) fait ainsi apparaître de nouvelles juridictions intellectuelles. Si de nouveaux axes de circulation contribuent à la reconfiguration d'une géographie des savoirs religieux, qu'en est-il des interactions entre ces juridictions et les canaux de diffusion de ces savoirs, académiques et vernaculaires? Seuls quelques contributeurs soulignent, par exemple, d'étroites et anciennes collaborations

14. I. Reader, "Japanese Studies of Pilgrimage », art. cité, p. 24.

15. A. Niedźwiedź, art. cité, p. 74-77.

16. G. Barna, art. cité. 
scientifiques entre la France et l'Italie, ou mentionnent encore l'impact de la French Theory sur la pensée anglo-saxonne, en Angleterre, en Amérique du Nord, au Mexique ou au Brésil.

\section{De l'immobilité à la mobilité, et retour}

Il n'est pas très étonnant, comme le soulignent Dionigi Albera et John Eade ${ }^{17}$, que durant la période 1950-1970, les anthropologues indianistes n'aient accordé que peu d'intérêt aux déplacements massifs de population engendrés par la pratique du pèlerinage; ils étaient plus concentrés sur les communautés territorialisées à l'intérieur des limites villageoises. Dans la quasi-totalité des contextes évoqués par les contributeurs, la plupart des recherches ne se sont que très tardivement focalisées sur cette dimension singulière du mouvement-déplacement, l'accent étant plutôt mis, à l'exception de quelques auteurs, sur la seule configuration des lieux de destination (églises, temples, mausolées soufis, etc.) et des pratiques rituelles qui s'y déroulent, au détriment des foyers de départ et de l'expérience sensible du voyage. Or, la pratique du pèlerinage sur de longues distances constituait en Inde, avant la modernisation des transports et l'essor du tourisme, la grande expérience du voyage dans une société relativement stable, parfois une occasion unique pour les pèlerins (notamment les femmes) de voyager au-delà de leurs territoires du quotidien. Le pèlerinage conférait dès lors un sens alternatif à l'expérience des déplacements de proximité ${ }^{18}$. Il ne faut cependant pas attendre la version anglosaxonne du "tournant de la mobilité », tel qu'il a été formulé par John Urry et consorts au début des années 2000, ou l'ouvrage de Simon Coleman et John Eade ${ }^{19}$, pour que le mouvement comme force de transformation soit pris en compte dans les études de pèlerinage.

Son importance est à rechercher, par exemple, dans le contexte japonais ${ }^{20}$ où les figures de l'itinérance (saints, moines, guides, promoteurs), par rapport à la sédentarité de la société paysanne, sont centrales en ce qu'elles orientent les pratiques locales au quotidien ou bien qu'elles structurent le pèlerinage emblématique de Shikoku entre les années 1950 et 1970. D'autres études soulignent l'intérêt dirigé vers l'itinérance ascétique en Inde (hindouisme) et au Pakistan (soufisme) dans ses rapports à la stabilité de mouvements religieux institutionnalisés (monastères, confréries), mais aussi en Russie où, contrairement à d'autres traditions religieuses, la vénération et la proximité avec la matérialité du divin prend le dessus sur le mouvement et la distance, comme c'est le cas des processions d'icônes ou de reliques, sortes de "pèlerinages inversés» qui participent au déplacement de la centralité du pouvoir patriarcal vers la périphérie ${ }^{21}$. Comment le mouvement rituel, continu ou intermittent, de ces figures d'itinérance est-il considéré dans

17. J. Eade, D. Albera, «Pilgrimage Studies in Global Perspective», in Albera D., Eade J. (eds.), New Pathways in Pilgrimage Studies..., op. cit., p. 3.

18. S. Coleman, "Do you believe in pilgrimage? Communitas, contestation and beyond", Anthropological Theory, 2-3, 2002, p. 364.

19. S. Coleman, J. Eade, op. cit.

20. I. Reader, "Japanese Studies of Pilgrimage ", art. cité.

21. S. Rock, art. cité, p. 56-57. 
différentes traditions religieuses? Et comment s'articule-t-il avec d'autres formes de mobilité humaine, qu'il s'agisse du pèlerinage, du tourisme ou de la migration? Une approche comparative de ces multiples formes permettrait certainement aux pèlerinologues de mieux participer au débat sur l'impératif de mobilité, celle-ci étant aujourd'hui hyper-valorisée par les pouvoirs publics comme par les laboratoires de recherche en sciences sociales, qui a essaimé dans les sociétés occidentales au tournant du $\mathrm{XxI}^{\mathrm{e}}$ siècle.

À l'inverse des premières études, les approches du pèlerinage comme « rituel cinétique », pour reprendre le vocable d'Edith Turner ${ }^{22}$, valorisent le mouvement et la mobilité rituelle dans l'espace au point de faire totalement disparaître les lieux de départ et d'arrivée, comme ce fut le cas d'ethnographies mobiles conduites, par exemple, dans les bus de pèlerins en Inde ou le long des chemins de Compostelle ${ }^{23}$. Mais l'impératif de mobilité ferait presque oublier que le mouvement ne peut s'envisager sans son pendant structural, l'immobilité ou la fixité à laquelle il ne convient pas d'associer forcément des valeurs positives ou négatives, elles-mêmes étant variables dans le temps des sociétés. Tout voyage, à vocation touristique et/ ou religieuse, ne se prépare-t-il pas dans la conscience immobile de celles et ceux qui l'imaginent ou le planifient, avant qu'il n'advienne et ne se réalise en pratique? Peu d'auteurs s'intéressent aux lieux de départ et aux individus qui jouent un rôle actif dans la formulation de projets de voyage rituel sans y participer euxmêmes, ainsi qu'aux préparatifs au pèlerinage et à ses conséquences sociologiques sur le foyer d'émission ${ }^{24}$. Une telle approche, qui mériterait d'être développée plus systématiquement, permet aussi de revisiter alors les notions de liminalité (Van Gennep) et de communitas (Turner) pour intégrer aux études de pèlerinage la question de l'individualisme. En Russie, par exemple, deux auteurs prééminents se positionnent très différemment dans le contexte de la spiritualité orthodoxe: Kormina relève un certain degré d'égalité parmi les pèlerins sans voir pour autant poindre de sentiment d'appartenance communautaire, chacun recherchant son propre salut individuel et ignorant ses co-voyageurs; Naletova voit au contraire dans le pèlerinage la formation de "communautés kénotiques» et dans l'étape liminaire de mobilité (travail et habitat en commun) un moyen de réintégration dans l'espace social du fait des nouvelles relations interindividuelles qui s'y nouent ${ }^{25}$. Ce dernier point est intéressant car les auteurs envisageant le pèlerinage sous l'angle de l'exceptionnalité et de la liminalité ne prennent pas toujours en compte le repositionnement des individus dans l'espace social du quotidien, et donc le changement de statut induit par l'expérience du voyage religieux dont l'accumulation ou la récurrence (capital spirituel) peut aussi démultiplier l'effet de prestige social à l'échelle locale et favoriser une forme d'ascension sociale symbolique.

22. V. Turner, E. Turner (eds.), Image and Pilgrimage in Christian Culture. Anthropological Perspectives, New York, Columbia University Press, 1978.

23. A. Gold, Fruitful Journeys: The Ways of Rajasthani Pilgrims, Berkeley, University of California Press, 1988; N. L. Frey, Pilgrim Stories. On and Off the Road to Santiago, Berkeley, University of California Press, 1998.

24. M. Sallnow, Pilgrims of the Andes: Regional Cults in Cusco, Washington, Smithsonian Institution Press, 1987; F. Fogel, «Des voyageurs immobiles. Pratiques communautaires autour du pèlerinage (Haute Égypte) », Théologiques, 15-1, 2007, p. 19-42.

25. S. Rock, art. cité, p. 60-61. 
Autre moment de bifurcation à l'aune du mouvement et du tournant de la mobilité, le développement des recherches articulant le pèlerinage avec les migrations et le tourisme. Nombreux sont les chapitres à évoquer la dimension circulatoire commune à ces formes de mouvement-déplacement et dont la frontière qui les séparait n'a cessé de se rétrécir. Ainsi se développe, depuis une quinzaine d'années, un certain nombre de notions composées telles que le tourisme religieux et patrimonial, le pèlerinage touristique, le tourisme vernaculaire, les catégories de pèlerins-touristes ou de touristes spirituels dans le cas des Nouveaux mouvements religieux, pour aborder la problématique du pèlerinage, des circulations et des rassemblements qu'il implique. Mais le pèlerinage peut-il s'étudier comme une migration? Quel a été l'apport des études migratoires, qui ont connu un important renouvellement, à l'analyse du pèlerinage et inversement? Les rapports entre pèlerinage, migration et tourisme s'analysent essentiellement aujourd'hui en décrivant la mobilité du religieux à travers les migrations, c'est-à-dire en évaluant l'impact d'un contexte migratoire sur la religiosité de migrants ou autres circulants ${ }^{26}$. Si le pèlerinage vers Israël a longtemps été décrit comme la métaphore du voyage vers la terre natale, des études plus récentes conduites par des sociologues et anthropologues soulignent l'importance des immigrants juifs sépharades qui, durant les années 1950-1960, ont importé d'Afrique du Nord des figures de sainteté locale, générant ainsi une sorte de «judaïsme périphérique » par rapport aux grands lieux saints à Jérusalem ${ }^{27}$. Ailleurs, au Mexique, les lieux de culte sont considérés par certains auteurs hispanophones comme des points d'ancrage dans un territoire circulatoire permettant aux individus de maintenir le lien avec leurs territoires d'origine ${ }^{28}$. La notion de «territoire circulatoire» employée ici n'est pas sans rappeler celle formalisée par le sociologue Alain Tarrius pour décrire les pratiques socio-spatiales des migrants autour de la Méditerranée, en dehors de toute considération d'appartenance religieuse ${ }^{29}$. Il serait alors intéressant d'engager un travail réflexif, à côté de l'analyse thématique des juxtapositions ou superpositions entre différentes formes de circulation (migratoire, rituelle, touristique), en étudiant les filiations intellectuelles, dans un cadre comparatif, qui relient des notions similaires utilisées en différents points du globe pour décrire les circulations religieuses, infra ou supranationales. Il convient d'ajouter aussi que le paradigme de la mobilité religieuse en Afrique du Sud n'est pas le pèlerinage mais plus simplement le voyage ${ }^{30}$, d'où l'importance de prendre en considération les conceptions émiques du mouvement-déplacement rituel, révélées de manière empirique.

26. S. Bava, S. Capone (éds.), "Migrations et transformations des paysages religieux ", Autrepart. Revue de sciences sociales au Sud, 56-4, 2010, p. 3-15.

27. N. Luz, N. Collins-Kreiner, «Studying Jewish Pilgrimage in Israel », in Albera D., Eade J. (eds.), International Perspectives on Pilgrimage Studies..., op. cit., p. 134-151.

28. A. A. Ros, art. cité.

29. A. Tarrius, "Territoires circulatoires et espaces urbains: différenciations des groupes migrants », Les Annales de la recherche urbaine, 59-60, 1993, p. 51-60. Pour un essai d'application de cette notion à l'espace du pèlerinage en contexte soufi, voir R. Delage, "L'espace du pèlerinage comme territoire circulatoire. Sehwan Sharif le long de l'Indus ", Cahiers d'Outre-Mer, 275, 2017.

30. S. du Plooy, "South(ern) African Journeys of Reverence», in Albera D., Eade J. (eds.), New Pathways in Pilgrimage Studies..., op. cit., p. 124-141. 
Enfin, le pèlerinage est de plus en plus associé au tourisme, en témoignent le nombre grandissant d'articles et d'ouvrages sur ce sujet ou encore la collection dédiée, Routledge Studies in Pilgrimage, Religious Travel and Tourism, dans laquelle ont été publiés les deux ouvrages recensés ici. Présentant des analogies structurelles certaines, ces deux formes de déplacement, à vocation religieuse et récréative, ont longtemps été opposées l'une à l'autre et étudiées dans des champs de recherche distincts. Il peut paraître surprenant que l'article d'Alphonse Dupront ${ }^{31}$ et son approche phénoménologique et comparative de l'acte pèlerin et du voyage touristique n'aient jamais été cités par les contributeurs. Qu'est-ce qui rapproche la figure du pèlerin de celles du migrant ou du touriste si ce n'est l'expérience du voyage ? Elles présentent en effet de nombreuses similitudes, tant au niveau des comportements individuels que des attitudes de consommation de l'ailleurs, jusqu'à parfois se confondre totalement, dans le contexte de la mondialisation et du passage à la postmodernité, comme cela a pu s'observer dans de nombreux lieux de culte et de pèlerinage dans le monde. L'un des exemples les plus représentatifs de cette association concerne la Malaisie péninsulaire ${ }^{32}$ où le pèlerinage à la Mecque est devenu depuis les années 1960 une pratique fortement régulée par l'État et un nombre limité d'agences de voyage officielles assume la charge exclusive de filtrer, sélectionner et former les futurs candidats au Hajj mais aussi d'orienter leurs parcours vers des destinations touristiques. Pèlerinage et tourisme peuvent également converger et se confondre dans le cadre de voyages rituels marqués par leur dimension commémorative ou patrimoniale, ce qui permet de réintégrer à l'analyse la mémoire et son instrumentation par la société, l’État et le politique.

\section{Mémoire, territoire et patrimonialisation}

Plusieurs auteurs mettent l'accent sur les rapports entre territoire et pèlerinage, en insistant sur le rôle du temps, de la temporalité et des images du passé (lieux, figures, évènements) dans la structuration de la mémoire collective, locale ou nationale. Les chercheurs mexicains furent parmi les premiers à accorder une grande importance à l'espace et au territoire ${ }^{33}$. Parmi eux, Velasco Toro voyait dans le lieu de culte l'expression d'une région dévotionnelle, lisible à travers sa composition multi-ethnique et l'imbrication de cosmogonies indigènes, une notion similaire à celle de «culte régional » développée plus tôt par Richard Werbner et réutilisée par sa fille Pnina Werbner dans ses travaux sur le soufisme sud-asiatique. Parallèlement, Alicia Barabas envisageait les pèlerinages comme marqueurs de territoires ethniques, autonomes et de forme réticulaire, à travers lesquels se reproduisent les relations sociales. Ces travaux étaient fortement influencés d'un côté, par les sciences sociales marxistes brésiliennes et, d'un autre côté, par certaines figures de l'anthropologie nord-américaine comme Alan Morinis ou encore

31. A. Dupront, «Tourisme et pèlerinage. Réflexions de psychologie collective », Communications, 10-1, 1967, p. 97-121.

32. Y. Seng-Guan, "Religious Pluralism and Pilgrimage Studies in West (Peninsular) Malaysia ", in Albera D., Eade J. (eds.), New Pathways in Pilgrimage Studies..., op. cit., p. 68-88.

33. A. A. Ros, art. cité. 
Michael Sallnow, dont le travail pionnier sur les cultes péruviens prenait en compte à la fois l'espace, le territoire et le paysage (plus largement l'écologie) selon une démarche phénoménologique d'analyse régionale ${ }^{34}$.

Dans les discours et récits véhiculés à travers la pratique du pèlerinage, de la part des institutions régulatrices ou des individus eux-mêmes, la mobilisation récurrente de références à un territoire, idéalisé ou contesté, qu'il s'agisse d'une terre ancestrale (quittée, perdue ou confisquée), d'une ancienne royauté, d'un pays mythique, etc. démontre que ces lieux de pèlerinage sont tout autant des points de convergence des pratiques sociales, rituelles et politiques à l'échelon local que des lieux d'attachement et d'identification pour les sociétés qui y projettent leurs aspirations diverses. Les pèlerinages sont dans bien des cas des moments d'exaltation de la mémoire nationale, construite dans une forme de continuité imaginée et réinventée à différents tournants de l'histoire politique des sociétés. Ce fut le cas en Pologne où, en partance pour Jasna Gora à Czetochowa, érigé au rang de "sanctuaire national " au cours du XIX ${ }^{e}$ siècle, les paysans du sud font systématiquement halte à Cracovie, ancienne capitale et ville patrimoniale devenue le lieu de condensation de l'histoire polonaise et de l'idéologie nationaliste ${ }^{35}$. Certains territoires nationaux sont parfois définis sur la base d'une idéologie religieuse exclusive. Le cas d'Israël est emblématique de ce processus de construction d'une mémoire collective nationale, alimentée et orientée par une idéologie sioniste et une conception de la «Terre sainte» qui lui est propre, à travers la sacralisation de sites archéologiques et de lieux de culte anciennement partagés ${ }^{36}$. Les grands lieux saints mais aussi des lieux de culte plus modestes sont réinvestis comme sites de mobilisation afin de légitimer l'autorité politique et spirituelle du peuple juif sur un territoire dont les frontières imaginées dépassent celles de son État-Nation. C'est sur le même mode que les sociétés voisines, palestinienne ou jordanienne ${ }^{37}$, légitiment leur emplacement et affirment leur différence en "vernacularisant» leurs topographies religieuses et en réactivant de nombreux cultes locaux. On retrouve des formes analogues de sacralisation du territoire national dans d'autres contextes comme l'Inde où les mouvements nationalistes hindous diffusent une idéologie en partie fondée sur la représentation (cartographique) de la "Déesse Inde ». Tous ces exemples illustrent des processus de réappropriation de lieux ou de territoires contestés dont l'une des modalités est la resacralisation du paysage et de la nature.

Comment s'articulent conceptuellement le lieu saint et le territoire ? S'ils condensent le passé et le présent, des imaginaires importés et des configurations vernaculaires, les lieux de culte comme les grands lieux de pèlerinage suggèrent l'existence de territoires plus vastes, réels ou mythiques, identitaires ou juridictionnels. Cette articulation symbolique, qui ne relève pas de la simple variation de l'échelle spatiale d'analyse, permet de décrire la nature des rapports du lieu au territoire à travers la figure de rhétorique de la synecdoque et l'idée de conden-

34. M. Sallnow, op. cit.

35. A. Niedźwiedź, art. cité.

36. N. Luz, N. Collins-Kreiner, art. cité.

37. E. Aubin-Boltanski, Pèlerinages et Nationalisme en Palestine: prophètes, héros et ancêtres, Paris, Éditions de l'EHESS, 2007; N. Neveu, «La sacralisation du territoire jordanien ", Archives de sciences sociales des religions, 151, 2010, p. 107-128. 
sation ${ }^{38}$. La contraction du temps long dans le temps présent invite également à se pencher, à la lumière du Memory boom et du tournant patrimonial en sciences sociales, sur le rôle de la mémoire collective dans la co-construction des lieux de pèlerinage et des territoires qu'ils expriment. En islam et dans l'ensemble des pays musulmans, la mise en patrimoine et les pratiques qui l'accompagnent, du Monde Arabe à l'Asie centrale jusqu'au sous-continent indien et au-delà, sont indissociables des vaqf ( fondation pieuse»), dont la nationalisation par l'État les extrait de la sphère privée du religieux. Les sites sont envisagés comme des ressources territoriales au service de politiques de patrimonialisation et de promotion de nouvelles formes de tourisme comme cela est le cas, par exemple, de sanctuaires soufis en perte de vitesse au Maghreb ${ }^{39}$. Dans une société laïque comme l'Australie, les lieux faisant l'objet de pèlerinages séculiers sont aussi bien des champs de bataille, des cimetières que des monuments aux morts érigés en mémoire des Première et Seconde Guerres mondiales ${ }^{40}$. Dans le premier cas, les programmes de rénovation visent la promotion d'une identité culturelle régionale ou nationale selon un mouvement orienté vers l'avenir. Dans le second, les commémorations sont des formes d'expression laïque des valeurs religieuses nationales. Ces actions de mobilisation de la mémoire (religieuse et historique) et de valorisation d'un héritage aboutissent toutes deux au développement de nouvelles formes de tourisme, religieux et/ou patrimonial, dépassant ainsi les oppositions binaires sacré-profane ${ }^{41}$ qui structuraient jusqu'à récemment un champ disjoint d'études sur le pèlerinage et le tourisme.

C'est donc bien dans un double mouvement de patrimonialisation et de reterritorialisation, associant diverses composantes du patrimoine, familial, foncier et rituel, que les évolutions du capital mémoriel d'une société peuvent s'observer et s'interpréter dans la mesure où le patrimoine constitue à la fois un enjeu social au niveau local (mémoire de classe), idéologique et politique (mémoire historique), voire diplomatique si l'on s'intéresse au déploiement des missions archéologiques internationales.

\section{Du paradigme de la contestation à la religion en partage}

Insistant sur le renouvellement des approches du pèlerinage, notamment dans ses rapports avec les phénomènes migratoires, le tourisme ou la patrimonialisation, Dionigi Albera John Eade notent également que la transition du modèle de la contestation vers celui des lieux saints partagés n'est pas encore achevée, en témoigne le nombre de travaux récents axés sur l'analyse des conflits et des discours

38. B. Debarbieux, «Le lieu, le territoire et trois figures de rhétorique », L'espace géographique, 2, 1995, p. 97-112; voir aussi M. Claveyrolas, R. Delage (éds.), Territoires du religieux dans les mondes indiens. Parcourir, mettre en scène, franchir, Paris, Éditions de l'EHESS, coll. «Purusartha », 2016. 39. K. Boissevain, art. cité.

40. A.-K. Hermkens, "Pilgrimage Studies in Oceania: Betwixt and Between National Concerns, Academic Trends and Local Ontologies ", in Albera D., Eade J. (eds.), New Pathways in Pilgrimage Studies..., op. cit., p. 106-123.

41. Sur ce point, voir aussi M. Claveyrolas, "The Amazement of the Ethnographer: Hindu Pilgrimage Beyond Sacred and Profane ", in Albera D., Eade J. (eds.), New Pathways in Pilgrimage Studies..., op. cit., p. 36-52. 
de revendication dans le contexte des rassemblements religieux, comme au Brésil ${ }^{42}$, au Mexique ou en Afrique du Sud. Aussi la phase de normalisation des sciences sociales du religieux à l'œuvre en Russie ou en Pologne depuis deux décennies s'est-elle traduite par une réorientation des études du pèlerinage, de l'analyse des mouvements contestataires ou de l'affirmation d'une identité nationale vers celle des pèlerinages ou processions rituelles comme outils performatifs à l'intérieur d'espaces multiconfessionnels. Mais ces deux modèles paradigmatiques, ainsi que celui de la mobilité, sont-ils incompatibles et doivent-ils forcément s'appliquer séparément? Au contraire, des publics très divers associés à différents types d'acteurs et d'institutions, religieux ou laïques, construisent ensemble le sens d'un lieu, au quotidien ou à travers le dispositif mobile et transitoire du pèlerinage, autant sur le mode de la dispute ou de la compétition que celui de la coopération, de la solidarité et de la négociation.

Les pèlerinages mixtes ou pluriconfessionnels ont retenu l'attention dans d'autres régions du monde que les pourtours méditerranéens ${ }^{43}$. En Malaisie, par exemple, le pouvoir colonial a contribué à l'émergence de la péninsule comme carrefour majeur des circulations marchandes et favorisé du même coup l'installation de migrants de confessions diverses. Certains complexes rituels traditionnels ont été sinisés par les dévots chinois, le culte des saints soufis y a été implanté par les marchands tamouls, des pèlerinages pluriconfessionnels autour de lieux de culte catholiques ou hindous s'y sont développés, sans toutefois faire l'objet de travaux anthropologiques approfondis ${ }^{44}$. L'Asie du Sud (surtout l'Inde et le Pakistan) a vu se développer très tôt, dès le $\mathrm{XIX}^{\mathrm{e}}$ siècle dans les récits des premiers orientalistes, une approche du soufisme comme foi commune, génératrice de mixité tolérante entre hindous et musulmans ${ }^{45}$. Plus récemment, Carla Bellamy qualifiait la culture sud-asiatique des sanctuaires soufis (dargah) de cosmopolite ${ }^{46}$ du fait de la grande mixité sociale, religieuse et culturelle que l'on peut y observer. Mais la coprésence affichée de publics variés (minorités, femmes, etc.), à l'occasion de rassemblements festifs, traduit-elle un niveau de mixité suffisamment élevé pour générer de nouvelles sociabilités cosmopolites? Les interactions sociales sont-elles de même intensité à l'intérieur et à l'extérieur de la société mobile du pèlerinage?

Invoquer le caractère traditionnellement inclusif du soufisme, une vision partiellement héritée de l'ethnographie coloniale, ne constitue pas un facteur explicatif suffisant. Il apparaît donc nécessaire de décrire les formes sociales et spatiales, ainsi que leur articulation à différentes échelles, d'une religiosité et d'une

42. C. A. Steil, «Studies of Catholicism and Pilgrimage in Brazil: Continuities and Ruptures over the Long-term ", in Albera D., Eade J. (eds.), New Pathways in Pilgrimage Studies..., op. cit., p. 162-180. 43. Voir notamment D. Albera, M. Couroucli (éds.), Religions traversées. Lieux saints partagés entre chrétiens, musulmans et juifs en Méditerranée, Arles, Actes Sud, 2009; D. Albera, "La mixité religieuse dans les pèlerinages. Esquisse d'une réflexion comparative ", Archives de sciences sociales des religions, 155, 2011, p. 109-129; I. Dépret, G. Dye (éds.), Partage du sacré. Transferts, dévotions mixtes, rivalités interconfessionnelles, Fernelmont, Éditions EME, 2012.

44. Y. Seng-Guan, art. cité.

45. M. Boivin, "Sufism, Pilgrimage and Saint Worship in South Asia », in Albera D., Eade J. (eds.), New Pathways in Pilgrimage Studies..., op. cit., p. 53-67.

46. C. Bellamy, The Powerful Ephemeral. Everyday Healing Practices in an Ambiguously Islamic Place, New Delhi, Permanent Black, 2011. 
sociabilité cosmopolites érigées dans le mouvement, non pas du point de vue de l'interpénétration de cosmologies ou en termes de convergences doctrinales mais à travers la description des pratiques de partage de l'espace public. Par sa fonction de médiation du rapport à l'altérité sociale et religieuse, le pèlerinage ne participe pas seulement à la reproduction des relations sociales ou à la consolidation des frontières internes à un groupe ; il est plus simplement facteur de repositionnement des individus et des groupes les uns par rapport aux autres, en d'autres termes facteur de perméabilisation des frontières symboliques de l'identité dans un contexte favorisant les expériences individuelles de butinage religieux mais également la mise en place de hiérarchies temporaires, par exemple entre les groupes de visiteurs et la population locale. Aussi les institutions gouvernementales en charge de l'administration des lieux saints soufis jouent-elles un rôle important de médiation et de régulation de ces rassemblements, en contrôlant l'accès à l'espace public et en encadrant les pratiques rituelles dans et aux abords d'un sanctuaire. En initiant des programmes de transformation architecturale (destruction et/ou rénovation) qui s'accompagnent parfois de l'effacement délibéré dans le paysage urbain de traces jugées non-conformes à la norme religieuse prescrite, l'État participe aussi à la transformation du rapport visuel et sensible des pèlerins à l'environnement local et donc du sens attribué au lieu saint.

C'est peut-être à travers ce type d'approches, avec d'un côté une économie politique soucieuse de l'articulation des échelles et des contextes de mobilité et, d'un autre côté, une science sociale du religieux recourant aux nouvelles méthodes visuelles et sensorielles, que les études de pèlerinage sont amenées à se renouveler.

Rémy Delage Centre d'Études de l'Inde et de l'Asie du Sud (EHESS-CNRS) delage.remy@gmail.com 